\section{DOXYCYCLINE INDUCED GASTRITIS AND OESOPHAGEAL ULCER}

Jin-Yu Chuang $^{1}$, I. Brown ${ }^{1,2}$, L. Conway ${ }^{3}$, M. Singh ${ }^{1}$

${ }^{1}$ Pathology Queensland, ${ }^{2}$ Envoi Pathology, Brisbane, and

${ }^{3}$ Cairns Base Hospital, Cairns, Australia

We report an unusual case of a 71-year-old female patient from Cairns Base Hospital who developed significant dysphagia following treatment with doxycycline for possible leptospirosis. Endoscopically, a lesion in the oesophagus thought to be 'oesophagitis desiccans superficialis' and what appeared to be a 'lichenoid lesion' in the upper stomach were seen. Microscopically, the oesophageal biopsies showed a benign ulcer. The gastric body and biopsies showed linear superficial gastric mucosal coagulative necrosis, focal cryptitis, crypt abscesses formation, associated regenerative changes together with coagulative necrosis of capillary walls. This is the second report of Doxycyline induced gastritis with concurrent oesophageal ulcer.

\section{TARGETED NEXT GENERATION SEQUENCING IN FFPE SAMPLES OF NSCLC}

W.A. Cooper $^{1,2,3}$, S. Kraitsek ${ }^{4}$, C.I. Selinger ${ }^{1}$, T. $\operatorname{Tran}^{1}$, M. Kohonen-Corish ${ }^{5,3,6}$, S.A. O'Toole ${ }^{1,2,5, *}$, B. Yu ${ }^{2,4, *}$

${ }^{1}$ Tissue Pathology and Diagnostic Oncology, Royal Prince Alfred Hospital, ${ }^{2}$ Sydney Medical School, University of Sydney, ${ }^{3}$ School of Medicine, University of Western Sydney, ${ }^{4}$ Medical Genomics, Royal Prince Alfred Hospital, ${ }^{5}$ Kinghorn Cancer Centre and Garvan Institute of Medical Research, and ${ }^{6}$ UNSW Medicine, Sydney, NSW, Australia; *joint senior authors

Background: With increasing clinical need for comprehensive molecular characterisation of non-small cell lung carcinoma (NSCLC), there is a great advantage in using targeted cancer panels and next generation sequencing (NGS) to simultaneously assess a wide range of mutations in multiple genes.

Aims and Methods: We aimed to assess the utility of the TruSeq Amplicon Cancer Panel that assesses 48 genes $(35 \mathrm{~kb}$ target regions) using the Illumina MiSeq platform in routine formalinfixed paraffin-embedded (FFPE) tumour samples from 31 resected lung adenocarcinomas. Results were compared to mutation analysis with the OncoCarta V.10 panel that covers hot spots in 19 genes using a mass spectrometry platform.

Results: 28 of 31 cases were suitable for NGS but 3 failed quality control. These 3 cases were all $>15$ years old while the rest were $<15$ years old. Using NGS, there were $12(42.9 \%)$ TP53 mutations, $11(39.3 \%)$ KRAS mutations, 9 (32.1\%) EGFR mutations and $2(7.1 \%)$ PIK3CA mutations. Mutations were also found in BRAF, ATM, PTEN, PDGFRA, ERBB4, MET, HRAS, STK11, HRAS, CTNNB1, NOTCH1 and SMARCB1 in one case each. All the $E G F R$ and KRAS mutations were also identified by mass spectrometry. Of the 3 cases that were unable to be assessed by NGS, mass spectrometry was performed and identified an $E G F R$ mutation and a BRAF mutation.

Conclusion: Targeted NGS performs well in FFPE samples of NSCLC and is feasible in a routine clinical setting.

\section{FATAL MENINGOENCEPHALITIS SECONDARY TO NEMATODE HALICEPHALOBUS GINGIVALIS INFECTION IN A HUMAN}

\author{
A. Crawford ${ }^{1}$, D. Dhatrak ${ }^{1}$, B. Koszyca ${ }^{1,2}$ \\ ${ }^{1}$ Department of Surgical Pathology, SA Pathology, and ${ }^{2}$ School \\ of Medical Sciences, The University of Adelaide, Adelaide, \\ South Australia, Australia
}

We present the case of a 73-year-old woman with a past history of rheumatoid arthritis for which she was being treated with etanercept and methotrexate and type 2 diabetes mellitus, who developed malaise, increasing confusion and unsteady gait over a period of four days, which rapidly progressed to coma. She was admitted to hospital and despite extensive investigation no cause for her symptoms of suspected meningoencephalitis could be identified. She died on day 9 after admission and permission was obtained for a post mortem examination. External examination of the brain showed congestion but the leptomeninges were clear. There was extensive maceration of the base of the brain and brain stem with necrosis of the temporal lobes, basal ganglia, anterior corpus callosum, right cerebral peduncle, cerebellum and sellar region. Histological examination revealed features of a meningoencephalitis characterised by a predominantly histiocytic inflammatory infiltrate within the leptomeninges and a perivascular chronic inflammation within the parenchyma. There was no granulomatous inflammation. Histology also demonstrated widespread infiltration of the brain by nematodes, which measured $250-300 \times 15-20$ microns, as well as eggs measuring $40-55 \times 20-25$ microns. The nematodes were present throughout the parenchyma but were predominantly seen within the Virchow-Robbin spaces and around blood vessels. They displayed a rhabditiform oesophagus with a corpus, isthmus and bulb characteristic of Halicephalobus gingivalis and this was confirmed on real time PCR of the large-subunit ribosomal DNA (LSU rDNA) from brain tissue and CSF. No nematodes were identified outside the central nervous system. This organism, also referred to as Micronema deletrix or Halicephalobus deletrix, is a neurotropic nematode usually isolated from decomposing organic matter and horse manure. Meningoencephalitis secondary to $H$. gingivalis in humans is rare with only five cases reported in the literature. The natural history is of a rapidly progressive meningoencephalitis with an invariably fatal outcome. Helminth infection and specifically $H$. gingivalis needs to be considered in the differential diagnosis of a rapidly progressive meningoencephalitis of unknown aetiology, particularly in immunosuppressed individuals. This case also highlights the need for further investigation into possible diagnostic tests in the ante mortem setting.

\section{CARDIAC HAEMANGIOMA: A CONTEMPORARY REVIEW OF 152 TUMOURS PUBLISHED BETWEEN 1996-2014}

\section{Andrew Dettrick}

Pathology Queensland, Sunshine Coast, Health Services Queensland, Department of Health, Nambour Hospital, Australia 